This document is the accepted manuscript version of the following article: Pieber, S. M., Kambolis, A., Ferri, D., Bhattu, D., Bruns, E. A., Elsener, M., ... Baltensperger, U. (2018). Mitigation of secondary organic aerosol formation of 1 og wood burning emissions by catalytic removal of aromatic hydrocarbons. Environmental Science and Technology. https://doi.org/10.1021/acs.est.8b04124

\title{
Mitigation of secondary organic aerosol formation of log wood burning emissions by catalytic removal of aromatic hydrocarbons
}

\author{
Simone M. Pieber ${ }^{1,2, *}$, Anastasios Kambolis ${ }^{3}$, Davide Ferri ${ }^{3}$, Deepika Bhattu ${ }^{1}$, Emily A. Bruns ${ }^{1}$, \\ Martin Elsener ${ }^{3}$, Oliver Kröcher, ${ }^{3,4}$, André S.H. Prévôt ${ }^{1}$, Urs Baltensperger ${ }^{1, *}$ \\ ${ }^{1}$ Paul Scherrer Institute, Laboratory of Atmospheric Chemistry, 5232 Villigen, Switzerland \\ ${ }^{2}$ Empa, Laboratory for Air Pollution and Environmental Technology, 8600 Duebendorf, Switzerland \\ ${ }^{3}$ Paul Scherrer Institute, Bioenergy and Catalysis Laboratory, 5232 Villigen, Switzerland \\ ${ }^{4}$ École Polytechnique Fédérale de Lausanne, 1015 Lausanne, Switzerland \\ "Correspondence: \\ Simone M. Pieber (imone.pieber@psi.ch), Urs Baltensperger (urs.baltensperger@psi.ch)
}

\begin{abstract}
Log wood burning is a significant source of volatile organic compounds including aromatic hydrocarbons (ArHC). ArHC are harmful, reactive in the ambient atmosphere, and important secondary organic aerosol (SOA) precursors. Consequently, SOA is a major fraction of the total organic aerosol emitted by log wood burning. ArHC reduction is thus critical in the mitigation of adverse health and environmental impacts of log wood burning. In this study, two Pt-based catalytic

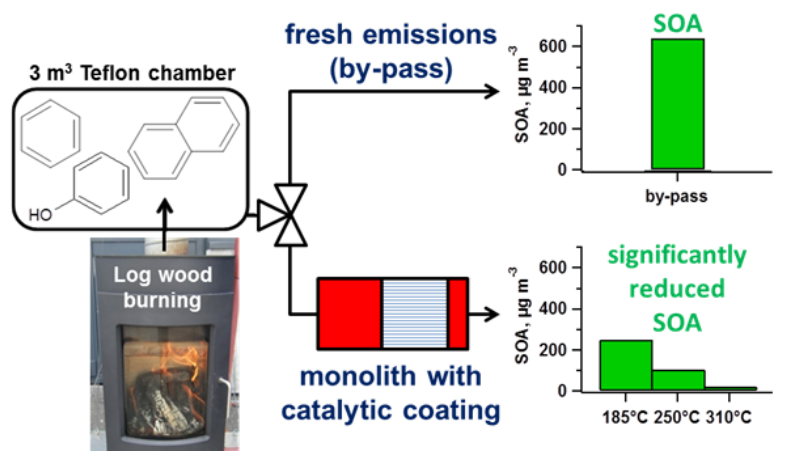
converters were prepared and tested for the mitigation of real-world log wood burning emissions, including ArHC and SOA (studied using a potential aerosol mass oxidation flow reactor), as well as toxic carbon monoxide (CO) and methane, a greenhouse gas. Substantial removal of mono- and polycyclic ArHC and in particular phenolic compounds was achieved with both catalysts operated at realistic chimney temperatures $\left(50 \%\right.$ conversion was achieved at 200 and $300^{\circ} \mathrm{C}$ for non-methane hydrocarbons in our experiments for $\mathrm{Pt} / \mathrm{Al}_{2} \mathrm{O}_{3}$ and $\mathrm{Pt} / \mathrm{CeO}_{2}-\mathrm{Al}_{2} \mathrm{O}_{3}$ respectively). The catalytically cleaned emissions exhibited a substantially reduced SOA formation already at temperatures as low as 185 to $310^{\circ} \mathrm{C}$, which substantially lowers the total PM burden of log wood burning. Thus, catalytic converters can effectively reduce primary and secondary log wood burning pollutants and thereby, their adverse health and environmental effects.
\end{abstract}

\section{Introduction}

Residential wood burning (WB) is often reputed to be a climate-neutral heating method due to its renewable nature and the emission of biogenic $\mathrm{CO}_{2}$, formed from carbon recently accumulated by plants. However, residential WB performed under non-ideal conditions is a known and substantial source of primary particulate matter (PM), toxic carbon monoxide (CO) and harmful volatile organic compounds (VOC) impacting air quality and human health. ${ }^{1-5}$

Primary PM in the submicron particle size range includes refractory material, such as elemental or black carbon (EC, BC) but may be dominated by non-refractory material such as primary organic aerosol (POA). For instance, ref. ${ }^{6}$ reported $\mathrm{POA} / \mathrm{BC}$ ratios of $2-14$ for $\log$ wood burning. In addition to primary PM emissions, formation of particulate secondary organic aerosol (SOA) from the atmospheric transformation of emitted reactive precursors is significant, reaching SOA/POA ratios of at least 3-7 in laboratory studies. $^{4,6-9}$

Harmful VOC emissions include aromatic, oxygenated aromatic and polycyclic aromatic hydrocarbons (ArHC). ${ }^{5}$ They are not only known to cause serious health issues in humans, through their carcinogenic effects (e.g. benzene is known to 
cause leukemia) or by deteriorating developmental, nervous, and heart and blood vessel systems ${ }^{10-12}$, but have also been identified as important SOA precursors in combustion emissions. ${ }^{5,9,13-15}$ Their relevance for ambient SOA formation is related to their reactivity towards hydroxyl radicals, and the formation of low-volatility compounds upon only few radical attacks, ${ }^{16},{ }^{17}$ which allows rapid condensation to the particle phase under typical atmospheric conditions. Additionally, incomplete WB leads to methane $\left(\mathrm{CH}_{4}\right)$ emissions ${ }^{18-20}$, which contribute relatively less to SOA formation owing to low SOA yields. ${ }^{21}$ However, methane emissions impact the environment by their high global warming potential.

Primary WB emissions (PM, CO and VOC including $\mathrm{CH}_{4}$ ) can be reduced by educational outreach, given the relevant influence of operator behavior $^{22-24}$, as well as by automating operation ${ }^{3}$ or improving burner technology. $6,8,25$ Switching from conventional log wood stoves to potentially cleaner automated systems or wood chip and pellet operation can be costly, might require incentives, and depends strongly on feedstock availability. While such measures will significantly reduce the emissions $^{26}$, they need to be combined with secondary pollutant abatement strategies in order to reduce the toxic and harmful pollutants to close-tozero levels for all appliance types in the future. Mechanical measures such as electrostatic precipitators may reduce primary $\mathrm{PM}$, but catalytic conversion is required for efficient $\mathrm{CO}$ and VOC removal $^{27}$, and the associated abatement of SOA. The use of catalytic converters has been explored at lab and pilot scale, focusing on the major primary pollutants including gases and primary particles ${ }^{27-}$ ${ }^{37}$, but studies lack any assessment of SOA abatement. As also the application of catalytic converters remains challenging ${ }^{31,}{ }^{37}$, further scientific studies are needed to establish their potential, especially when considering trace pollutants.

Here, we demonstrate simultaneous and significant reduction of harmful $\mathrm{CO}$, aromatic hydrocarbons, and SOA (through the removal of its precursors) from log wood stove emissions using Pt-based honeycomb catalytic converters.

\section{Material and Methods}

Wood burning (WB) experiments. Our appliance ${ }^{4,5,9}$ was fueled with dry beech wood (Fagus sylvatica). The emissions were sampled from the chimney over the whole burning cycle using a heated ejector diluter $\left(150^{\circ} \mathrm{C}\right)$ and injected into a custom-built $3 \mathrm{~m}^{3}$ Teflon chamber, made of $125 \mu \mathrm{m}$ thick collapsible DuPont Teflon fluorocarbon film (FEP) type 500A, Foiltec $\mathrm{GmbH}$, Germany. Dilution air was provided by a pure air generator system, which we described previously. ${ }^{13,38}$ The obtained well-mixed feed was diluted further with humidified clean air in the Teflon chamber and then passed alternately through a quartz glass reactor $^{39}$ containing a catalytic monolith at a flow rate of $5 \pm 1 \mathrm{~L} \mathrm{~min}^{-1}$ (gas hour space velocity, GHSV $=135 \mathrm{~L} \mathrm{~g}^{-1} \mathrm{~h}^{-1}$ ) and a by-pass line $\left(80^{\circ} \mathrm{C}\right)$ as shown in Figure S1. Behind the quartz glass reactor or by-pass line, the feed was diluted with clean dry air to provide suitable concentrations for the downstream analytical instruments, listed in Table S1, and fed to an oxidation flow reactor during photochemistry experiments (described below). The monolith was actively heated, and progressively set from 600 to $100^{\circ} \mathrm{C}$. Each temperature set-point was held for at least $5 \mathrm{~min}$ once the temperature stabilized. The quartz glass reactor was equipped with two $1 \mathrm{~mm}$ thermocouples positioned up- and downstream the monolithic catalyst. Compared to lab scale experiments $^{39}$, ceramic beads were not used upstream of the monolith in order to avoid particle losses, which caused, however, a large discrepancy between the temperature measurements up- and downstream and the actual set-point of the monolith (Figure S2). Therefore, we provide the set-point temperature. The catalysts were exposed to WB emissions containing $\mathrm{CO}_{2}$ (3400-10500 ppm), $\mathrm{CO}$ (360-1100 ppm), $\mathrm{CH}_{4}(65-220 \mathrm{ppm})$, non-methane hydrocarbons (NMHC, 80-260 ppmC) including aromatic hydrocarbons (ArHC, $70-320 \mathrm{ppmC}$ ), $1-3$ vol\% $\mathrm{H}_{2} \mathrm{O}$ and refractory as well as non-refractory primary aerosol particles. The modified combustion efficiency (MCE) determined as the ratio of $\mathrm{CO}_{2}$ to $\left(\mathrm{CO}+\mathrm{CO}_{2}\right)$ was 0.89-0.93 across all experiments. Feed concentrations are reported in Table 1 and Table 2, and refer to averages $\pm 1 \mathrm{SD}$ of the concentrations determined from by-pass measurements conducted 
at start, end and intermediate to selected temperature set-points. Catalytic conversion rates and pollutant reductions were calcualted by comparison of catalytically cleaned emissions to by-pass measurements of the corresponding experiment. Feed concentrations were subject to burn-to-burn variability as well as deliberate adjustments of the dilution factor in the Teflon chamber. Each experiment was conducted with emissions from a separate burn and Teflon chamber filling, experiment nomenclature is detailed below. Typically we conducted one experiment per day. The Teflon chamber was cleaned at the end of each experiment by injection of humidified air, ozone $\left(\mathrm{O}_{3}, 1 \mathrm{ppm}\right)$ and exposure to UV lights for 1 hour. Thereafter, it was flushed with dry clean air over night and refilled to about one third of its volume with humidified clean air before the next experiment.

Catalyst preparation. The catalyst powders $\left(\mathrm{Pt} / \mathrm{Al}_{2} \mathrm{O}_{3}\right.$ and $\left.\mathrm{Pt} / \mathrm{CeO}_{2}-\mathrm{Al}_{2} \mathrm{O}_{3}\right)$ were obtained by wet impregnation of chloroplatinic acid $\left(\mathrm{H}_{2} \mathrm{PtCl}_{6}\right.$, Fluka) on $\gamma-\mathrm{Al}_{2} \mathrm{O}_{3}(200 \mu \mathrm{m}$; Puralox SCCa150/200, Sasol) and $\mathrm{CeO}_{2}-\mathrm{Al}_{2} \mathrm{O}_{3}\left(30 \mathrm{wt} \% \mathrm{CeO}_{2}\right)$, which was prepared by deposition-precipitation $\left(\mathrm{Ce}\left(\mathrm{NO}_{3}\right)_{3} \cdot 6 \mathrm{H}_{2} \mathrm{O}\right.$ on the same $\left.\gamma-\mathrm{Al}_{2} \mathrm{O}_{3}\right)$. The final $\mathrm{Pt}$ content was ca. $1.3 \mathrm{wt}^{\mathrm{O}} \%$ as determined by elemental analysis. After drying overnight at $90^{\circ} \mathrm{C}$, the powders were calcined in static air at $500^{\circ} \mathrm{C}$ for $2 \mathrm{~h}$. Cordierite monoliths (cell density $=600$ cells per square inch; length $=77 \mathrm{~mm}$, diameter $=20 \mathrm{~mm}$, Figure S3) were dip coated in an aqueous slurry of the catalyst powder and a colloidal $\mathrm{Al}_{2} \mathrm{O}_{3}$ binder (Disperal, Sasol), calcined at $600^{\circ} \mathrm{C}$ for $6 \mathrm{~h}$, and then aged in $10 \mathrm{vol} \% \mathrm{H}_{2} \mathrm{O} / \mathrm{N}_{2}$ at $600^{\circ} \mathrm{C}$ for $10 \mathrm{~h}$ in a tubular quartz reactor. Monoliths were placed in a quartz glass reactor $^{39}$ for the experiments as described above.

Catalyst stability. We determined methane $\left(\mathrm{CH}_{4}\right)$ conversion rates on the catalytic monoliths using synthetic reactor feeds in the lab before and after exposure to WB emissions (section $\mathrm{S} 2.1$ provides the conditions). As shown in Figure S3, exposure to WB emissions left deposits indicated by a change in monolith surface color. Regardless of deposits, we did not observe any adverse effect on the $\mathrm{CH}_{4}$ conversion. While this indicates good stability, further assessment in long-term exposure experiments is suggested.
Photochemistry experiments. Secondary organic aerosol (SOA) formation was simulated using a potential aerosol mass oxidation flow reactor (OFR), ${ }^{15,38}$ which has been successfully applied to WB emissions earlier. ${ }^{25,38,40}$ The OFR consisted of a $0.015 \mathrm{~m}^{3}$ cylindrical glass chamber. It contained two low-pressure mercury UV lamps, each with discrete emission lines at 185 and $254 \mathrm{~nm}$, for hydroxyl radical generation from in-situ generated $\mathrm{O}_{3}(8 \mathrm{ppm})$ and $\mathrm{H}_{2} \mathrm{O}$ (15-20\% relative humidity), which was provided via a Nafion humidifier. Butanol-D9 was injected separately into the OFR and used to determine the hydroxyl radical concentration. ${ }^{41}$ The plug flow residence time was 100 s. SOA was determined as the organic particulate mass increase when OFR UV lamps were switched on. SOA of fresh emissions (i.e. emissions fed into the OFR through the by-pass line) was compared to that of catalytically cleaned emissions using $\mathrm{Pt} / \mathrm{Al}_{2} \mathrm{O}_{3}$ at $185^{\circ} \mathrm{C}$, and $\mathrm{Pt} / \mathrm{CeO}_{2}-$ $\mathrm{Al}_{2} \mathrm{O}_{3}$ at $185,200,250$ and $310^{\circ} \mathrm{C}$. OFR experiments were conducted at a median hydroxyl radical concentration of $1.1 \times 10^{9}$ molecules $\mathrm{cm}^{-3}$ (fresh emissions) and $1.5 \times 10^{9}$ molecules $\mathrm{cm}^{-3}$ (catalytically cleaned emissions). This corresponds to a median atmospheric reaction time of 29 hours (fresh) and 38 hours (catalytically cleaned, Table S2), assuming a mean ambient hydroxyl radical concentration of $1 \times 10^{6}$ molecules $\mathrm{cm}^{-3}$ following ref. $^{42}$. Recent publications presented in depth characterization operating conditions of OFRs ${ }^{43-46}$ and loss estimates of low-volatility compounds within the system. ${ }^{47}$ However, the here presented experiments were conducted before these assessments were available. Operating conditions were instead in line with previous experiments ${ }^{15,38}$ that yielded good agreement with smog chambers (considered to provide representative atmospheric processing in the past). The newer literature ${ }^{43-47}$ suggests that the here presented OFR experiments were conducted at an external hydroxyl radical reactivity $\left(\mathrm{OHR}_{\text {external }}\right)$ of 400-1350 s $\mathrm{s}^{-1}$ for fresh emissions. Experiments with catalytically cleaned emissions were more diluted $\left(\mathrm{OHR}_{\text {external }}: 2 \mathrm{~s}^{-1}\right.$ at $310^{\circ} \mathrm{C}, 230 \mathrm{~s}^{-1}$ at $250^{\circ} \mathrm{C}$ and $300 \mathrm{~s}^{-1}$ at $185^{\circ} \mathrm{C}$ ). Model results for photolysis by UV and a discussion of the influence of nitrogen oxides are presented in SI section S3. 
Instrumentation and data processing. Methane $\left(\mathrm{CH}_{4}\right), \mathrm{CO}$ and $\mathrm{CO}_{2}$ were measured using a cavity ring-down spectrometer (CRDS, Picarro). NMHC was calculated as the difference from flame ionization detection (FID, Horiba) of total hydrocarbons (THC) and $\mathrm{CH}_{4}$. We observed interference in the measurement at high ratios of $\mathrm{CH}_{4} / \mathrm{NMHC}$. Therefore, at high catalytic conversion of NMHCs $(>0.5)$ and in the presence of the yet initial $\mathrm{CH}_{4}$ concentration, the CRDSdetermined $\mathrm{CH}_{4}$ signal was used to calculate the NMHC. VOCs were characterized by a proton transfer reaction time-of-flight mass spectrometer ${ }^{48}$, 49 (PTR-ToF-MS; PTR-TOF-8000, Ionicon) using hydronium ions as the primary reagent. PTR-TOF8000 settings, data processing, ion identification and quantification were as described earlier. 5,9 Relevant ions and concentration levels are given in Table 2. The organic particulate matter (PM) was characterized using an aerosol mass spectrometer (HR-ToF-AMS, Aerodyne) equipped with a $\mathrm{PM}_{1}$ aerodynamic inlet lens. ${ }^{50}$ HR-ToF-AMS calibrations and data processing were as described earlier. ${ }^{9,}{ }^{51}$ A relative ionization efficiency of 1.4 (ref. ${ }^{51}$ ) and an experimentally determined collection efficiency $(0.6 \pm 0.1$, Table S2/Figure S11) were applied to organic PM. Data were corrected for gas-phase $\mathrm{CO}_{2}$ by measurements of particle-free emissions. Contribution of nitrate salts was negligible and no further $\mathrm{CO}_{2}{ }^{+}$correction was required. ${ }^{52}$

Experiment Nomenclature. We conducted experimental sets as described in the following:

- E3-E10 denote WB experiments throughout the manuscript. E5 $\left(\mathrm{Pt} / \mathrm{Al}_{2} \mathrm{O}_{3}\right)$ and $\mathrm{E}$ 3, E7, E8, E9 $\left(\mathrm{Pt} / \mathrm{CeO}_{2}-\mathrm{Al}_{2} \mathrm{O}_{3}\right)$ refer to catalytic cleaning of primary $\mathrm{WB}$ emissions.

- E6 $\left(\mathrm{Pt} / \mathrm{Al}_{2} \mathrm{O}_{3}\right)$, and E4, E10 $\left(\mathrm{Pt} / \mathrm{CeO}_{2}-\mathrm{Al}_{2} \mathrm{O}_{3}\right)$ denote photochemistry (SOA) experiments. These were conducted as add-on to E5, and E3, E9 respectively. For E6, and E4, E10 a higher dilution ratio was deployed in the Teflon chamber in order to reduce concentration levels for the subsequent OFR experiments. Catalytic conversion of primary WB emissions was therefore re-determined. We present the $\mathrm{CO}$ conversion for comparison in Figure S6.

- Catalyst stability was monitored by determining $\mathrm{CH}_{4}$ conversion using synthetic reactor feeds in the lab before (E1, E2) and after (E11, E12) exposure to WB emissions. Details are provided in SI section S2.1.

Table 1. Gas phase concentrations in the WB emissions feed (average concentration in Teflon chamber).

\begin{tabular}{|c|c|c|c|c|c|c|c|c|c|c|}
\hline $\begin{array}{l}\text { Expt. } \\
\text { No. } \\
\end{array}$ & Catalyst & $\begin{array}{l}\text { Expt. } \\
\text { Type }\end{array}$ & $\begin{array}{c}\text { Feed } \\
\text { L } \text { min }^{-1} \\
\end{array}$ & $\begin{array}{c}\mathrm{H}_{2} \mathrm{O} * \\
\% \\
\end{array}$ & $\begin{array}{c}\mathrm{CO}_{2} \\
\text { ppmC }\end{array}$ & $\begin{array}{c}\mathrm{CO}^{*} \\
\text { ppmC }\end{array}$ & $\begin{array}{l}\mathrm{CH}_{4}{ }^{*} \\
\text { ppmC }\end{array}$ & $\begin{array}{l}\mathrm{CH}_{4} * * \\
\operatorname{ppmC} \\
\end{array}$ & $\begin{array}{c}\text { NMHC** } \\
\text { ppmC }\end{array}$ & $\begin{array}{l}\text { THC** } \\
\text { ppmC }\end{array}$ \\
\hline E5 & $\mathrm{Pt} / \mathrm{Al}_{2} \mathrm{O}_{3}$ & primary WB & $\sim 4-6$ & $1 \%$ & 6000 & 450 & 80 & 85 & $90(95)$ & 175 \\
\hline E6*** & $\mathrm{Pt} / \mathrm{Al}_{2} \mathrm{O}_{3}$ & WB SOA & $\sim 4-6$ & $1 \%$ & 4000 & 360 & 65 & 70 & $80(85)$ & 150 \\
\hline E3 & $\mathrm{Pt} / \mathrm{CeO}_{2}-\mathrm{Al}_{2} \mathrm{O}_{3}$ & primary WB & $\sim 4-6$ & $1 \%$ & 6500 & 700 & 130 & 130 & $160(160)$ & 290 \\
\hline $\mathrm{E} 4 * * *$ & $\mathrm{Pt} / \mathrm{CeO}_{2}-\mathrm{Al}_{2} \mathrm{O}_{3}$ & WB SOA & $\sim 4-6$ & $1-3 \%$ & 5000 & 530 & 100 & 100 & $120(120)$ & 220 \\
\hline E7 & $\mathrm{Pt} / \mathrm{CeO}_{2}-\mathrm{Al}_{2} \mathrm{O}_{3}$ & primary WB & 5.5 & $1 \%$ & 8500 & 1000 & 200 & 190 & $260(250)$ & 450 \\
\hline E8 & $\mathrm{Pt} / \mathrm{CeO}_{2}-\mathrm{Al}_{2} \mathrm{O}_{3}$ & primary WB & 5.5 & $1 \%$ & 3400 & 400 & 75 & 75 & $105(105)$ & 180 \\
\hline E9 & $\mathrm{Pt} / \mathrm{CeO}_{2}-\mathrm{Al}_{2} \mathrm{O}_{3}$ & primary WB & 5.5 & $1 \%$ & 10500 & 1100 & 220 & 220 & $250(250)$ & 470 \\
\hline $\mathrm{E} 10 * * *$ & $\mathrm{Pt} / \mathrm{CeO}_{2}-\mathrm{Al}_{2} \mathrm{O}_{3}$ & WB SOA & 5.5 & $1 \%$ & 3700 & 380 & 75 & 75 & $85(85)$ & 160 \\
\hline
\end{tabular}

* Measurements based on cavity ring-down spectrometry (CRDS, Picarro). **Measurements based on flame ionization detection (FID) for THC and $\mathrm{CH}_{4}$, with the difference yielding the NMHC. NMHC values in brackets give the calculated NMHC as the difference between FID THC and $\mathrm{CRDS} \mathrm{CH}_{4} .{ }^{* * *} \mathrm{E} 6$ and E4, E10 are OFR (i.e. photochemistry/SOA) experiments during which the fresh primary WB emissions were also catalytically cleaned; E5 and E3, E7, E8, E9 are fresh emissions experiments w/o subsequent SOA formation. Note that all concentration levels here refer to the feed for the catalytic converters; emissions were diluted at least 8 -fold before being fed to instrumentation/OFR.

\section{Results and Discussion}

Primary gaseous emissions. Catalytic conversion of $\mathrm{CO}$, non-methane hydrocarbons (NMHC), specific volatile organic compounds (VOC) and methane was determined in wood burning (WB) emissions. Both tested catalysts were effective for the abatement of these species. They displayed distinctly different behavior in their temperaturedependent conversion curves. 

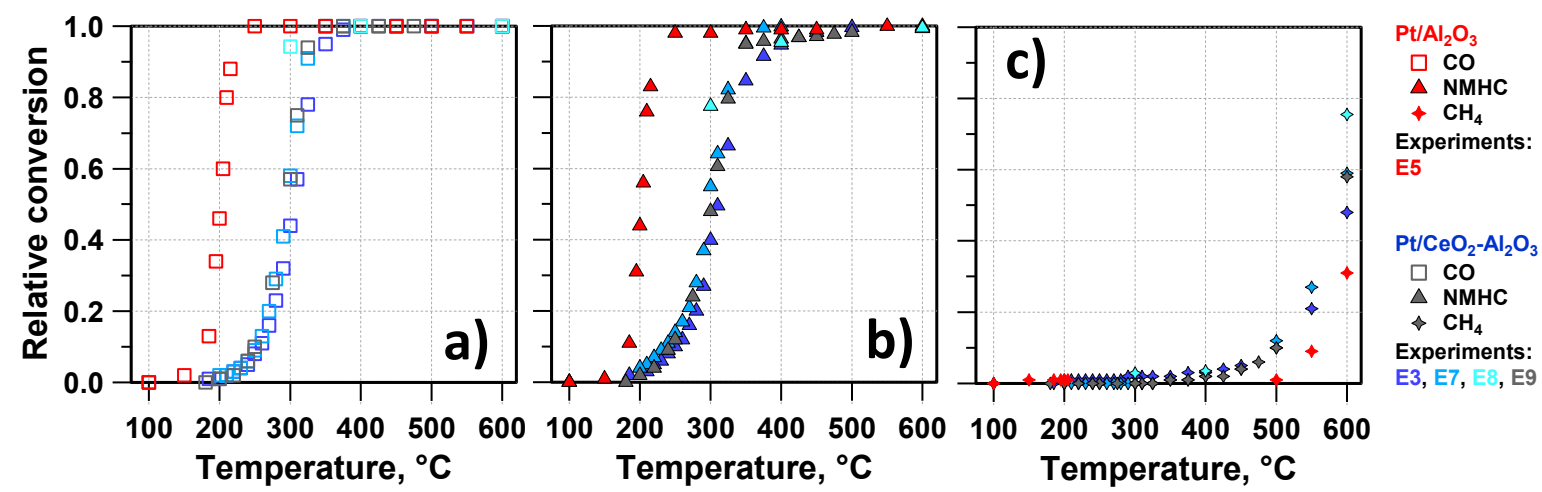

Figure 1. Catalytic conversion of a) $\mathrm{CO}$, b) $\mathrm{NMHC}$ and c) methane $\left(\mathrm{CH}_{4}\right)$ on $\mathrm{Pt} / \mathrm{Al}_{2} \mathrm{O}_{3}$ and $\mathrm{Pt} / \mathrm{CeO}_{2}-\mathrm{Al}_{2} \mathrm{O}_{3}$ in wood burning (WB) emissions. E5 and E3, E7, E8, E9 denote different experiments using fresh emissions (Table 1).

Significant removal of $\mathrm{CO}$ and $\mathrm{NMHC}$ was achieved at temperatures representative of startup and operation of wood appliances. ${ }^{28}$ The temperature of $50 \%$ conversion $\left(T_{50}\right)$ was significantly different between the two catalysts, but equal for $\mathrm{CO}$ and $\mathrm{NMHC}$ for a given catalyst. $\mathrm{Pt} / \mathrm{Al}_{2} \mathrm{O}_{3}$ provided better conversion at lower temperature $\left(T_{50}, 200^{\circ} \mathrm{C}\right)$ than $\mathrm{Pt} / \mathrm{CeO}_{2}-\mathrm{Al}_{2} \mathrm{O}_{3}\left(T_{50}\right.$, $300^{\circ} \mathrm{C}$ ). Both systems yielded a conversion $\geq 95 \%$ at $400^{\circ} \mathrm{C}$ (Figure 1). Methane $\left(\mathrm{CH}_{4}\right)$ is characterized by a relatively low reactivity in the atmosphere and generally in chemical reactions, which makes its catalytic abatement more challenging. ${ }^{53}$ While palladium is considered generally more efficient, platinum-based catalysts offer more resistance to sulfur. In our experiments, methane required higher reaction temperatures than $\mathrm{CO}$ and $\mathrm{NMHC}$, yet, both catalysts reached significant conversion at $600^{\circ} \mathrm{C}$. In contrast to our observations for $\mathrm{CO}$ and $\mathrm{NMHC}, \mathrm{Pt} / \mathrm{CeO}_{2}-\mathrm{Al}_{2} \mathrm{O}_{3}$ exhibited a significantly enhanced methane conversion between 400 and $600^{\circ} \mathrm{C}$ compared to $\mathrm{Pt} / \mathrm{Al}_{2} \mathrm{O}_{3}$. $\quad \mathrm{CeO}_{2}$ clearly facilitated methane oxidation, which is often attributed to the improved redox properties of the catalyst. ${ }^{54,55}$

The NMHC emissions were dominated by benzene (44-234 ppmC) and contained also naphthalene (8-40 ppmC) and phenolic compounds (8.6-23 ppmC) along with styrene (1.4-4.6 ppmC) and oxygenated aromatic or unsaturated oxygenated compounds, e.g., benzaldehyde (1.0$4.1 \mathrm{ppmC})$ and furans (1.2-3.6 ppmC). Overall, the composition (Table 2/Figure 3a) was in line with our previous findings. ${ }^{5} 9$ Concentration levels represented real-world stove operation, albeit our emissions correspond to relatively poor combustion conditions (indicated by the modified combustion efficiency, MCE, of 0.89-0.93). Both catalysts significantly removed aromatic hydrocarbons (ArHC) from WB emissions (Figure 2).

Functionalized molecules, such as phenolic ArHC (phenol, methylphenol, dimethylphenol), and also ArHC with carbonyl functions (e.g. benzaldehyde) were removed in significant amounts already at lower temperatures than their non-functionalized analogues (monocyclic and polycyclic ArHC). The abatement was nearly complete at set-point temperatures as low as $185^{\circ} \mathrm{C}$ for phenolic compounds. We could not assess decisively whether the losses on the ceramic monolith and on the $\mathrm{Al}_{2} \mathrm{O}_{3}$, which can act as coldtraps, contributed to the removal of phenolic compounds below $185^{\circ} \mathrm{C}$ (Figure S5). Nevertheless, the removal either by the temporary storage on cold-surfaces (and catalytic conversion once temperatures are sufficiently high) or by the direct catalytic abatement at low temperature is beneficial to reduce the health impact of the emissions, and had substantial effects on the reduction of the SOA potential (see below).

Various non-oxygenated ArHC of increasing molecular weight such as benzene, toluene, styrene and naphthalene exhibited temperature dependent conversion. While $\mathrm{Pt} / \mathrm{Al}_{2} \mathrm{O}_{3}$ converted these components and $\mathrm{CO}$ simultaneously, $\mathrm{Pt} / \mathrm{CeO}_{2}-\mathrm{Al}_{2} \mathrm{O}_{3}$ discriminated between different ArHC (Figure 2). It exhibited considerably higher activity for the conversion of more reactive compounds. This is reflected in the varied $T_{50}$ in the series. Benzene was oxidized in 
the same temperature regime as CO. Molecules with higher reactivity such as polycyclic ArHCs (e.g., naphthalene), or unsaturated functionalities (e.g., styrene) converted at lower temperatures than more stable, less reactive molecules (e.g., toluene and benzene; in line with their atmospheric reaction rate constant towards hydroxyl radicals $\left(k_{\mathrm{OH}}\right)$, see Table 2 and Figure 2). Typically, higher temperatures $\left(+20\right.$ to $+100^{\circ} \mathrm{C}$ depending on the molecule) were needed with $\mathrm{Pt} / \mathrm{CeO}_{2}-\mathrm{Al}_{2} \mathrm{O}_{3}$. Nevertheless, ArHCs were completely removed from the emissions by both catalysts at representative exhaust gas temperatures of $220^{\circ} \mathrm{C}$ $\left(\mathrm{Pt} / \mathrm{Al}_{2} \mathrm{O}_{3}\right)$ and $350^{\circ} \mathrm{C} \quad\left(\mathrm{Pt} / \mathrm{CeO}_{2}-\mathrm{Al}_{2} \mathrm{O}_{3}\right)$ demonstrating feasibility of the catalytic abatement.

SOA precursor identification and quantification ("SOA-22 precursors"). The species listed in Table 2 were identified as important precursors for SOA formation in wood burning emissions by an earlier study. ${ }^{9}$ Here we refer to the collection of these species as the sum parameter "SOA-22 precursors". The relative contribution of each species to the total available gas-phase mass as determined by PTR-ToF-MS is presented in Figure 3a. The sum of SOA-22 precursors accounted for $97 \pm 0.5 \%$ (based on mass in $\mu \mathrm{g} \mathrm{m}^{-3}$ ) of all potential SOA precursors identified in our fresh emissions. Henceforth, SOA-22 are used as a surrogate for all SOA precursors in our study. The largest fraction to the SOA-22 gas-phase mass was contributed by benzene (58-72\%), naphthalene (11-12\%), and phenol (6-9\%), as displayed in Figure 3a.

In order to estimate the fraction of relevant SOA forming carbon mass to the total available gaseous organic compounds the SOA-22 precursor fraction was compared to the total PTR-ToF-MS derived organic and carbon mass, as well as the FID-based carbon mass. The SOA-22 precursors accounted for $49 \pm 9 \%$ of the total PTR-ToF-MS detectable organic gas-phase mass $\left(\mu \mathrm{g} \mathrm{m}^{-3}\right)$, which corresponded to $65 \pm 8 \%$ if considering the carbon fraction $\left(\mu \mathrm{gC} \mathrm{m} \mathrm{m}^{-3}\right)$. The remainder $(51 \%$ and $35 \%$ respectively) was dominated by small oxygenated organic species, such as e.g. methanol and acetic acid, as also observed in earlier experiments. ${ }^{5,9}$ These compounds do not significantly contribute to SOA formation during atmospheric oxidation owing to their small carbon backbone.
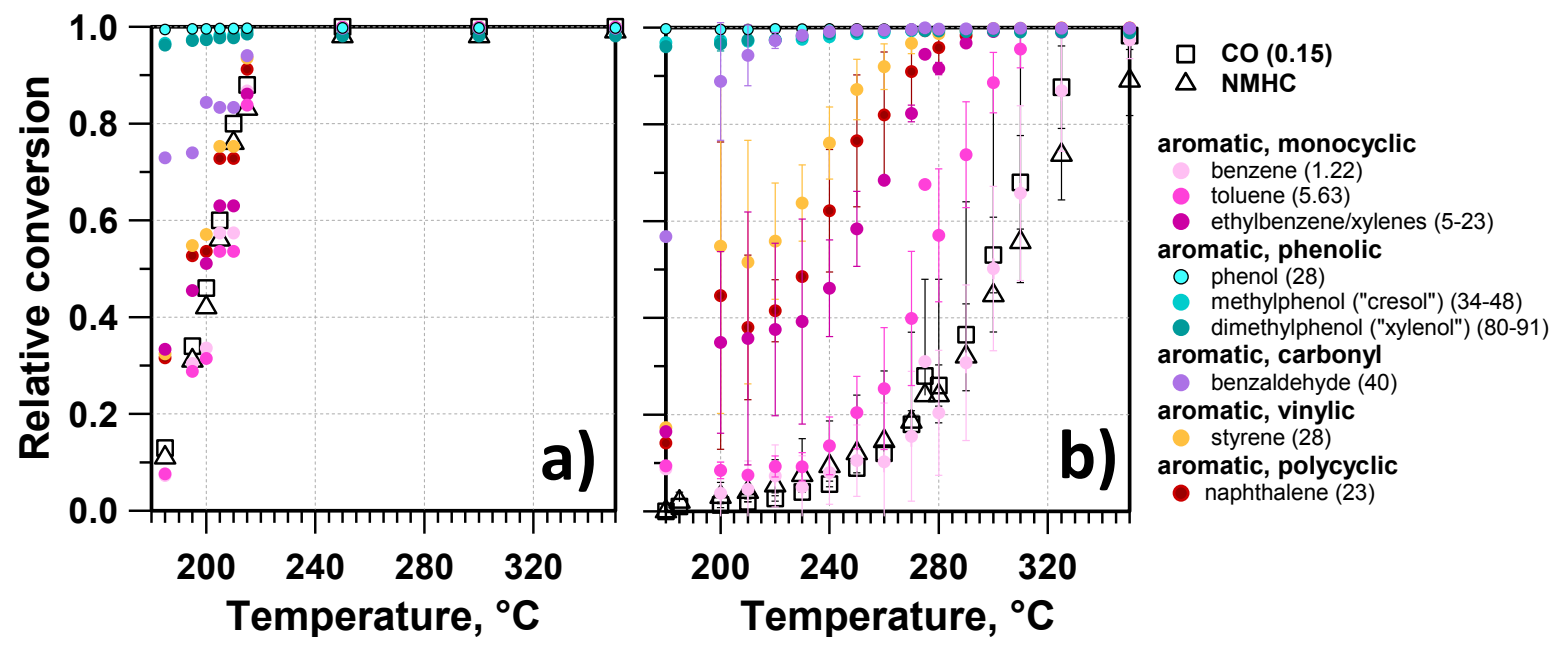

Figure 2. Catalytic conversion of specific aromatic hydrocarbons. a) $\mathrm{Pt} / \mathrm{Al}_{2} \mathrm{O}_{3}$ (E5), b) $\mathrm{Pt} / \mathrm{CeO}_{2}-\mathrm{Al}_{2} \mathrm{O}_{3}$ (average \pm 1 standard deviation of E3, E7, E8 and E9). Losses of compounds are discussed in Figure S5. CO and NMHC are displayed for comparison. Numbers in brackets in the legend indicate atmospheric hydroxyl radical rate constants $\left(10^{-12} \mathrm{~cm}^{3}\right.$ molecules $\mathrm{s}^{-1} \mathrm{~s}^{-1}$, Table 2$)$. 
Table 2. SOA-22 precursors and relative concentrations in catalyst feed.

\begin{tabular}{|c|c|c|c|c|c|c|c|c|c|c|c|}
\hline \multirow[t]{2}{*}{$\begin{array}{l}\text { Structural } \\
\text { assignment }^{5,9}\end{array}$} & \multirow[t]{2}{*}{$\begin{array}{l}\text { Compound } \\
\text { class }\end{array}$} & \multirow[t]{2}{*}{$\begin{array}{l}m / z \\
\text { see }^{\text {a) }}\end{array}$} & \multirow[t]{2}{*}{$\begin{array}{l}\text { Protonated } \\
\text { ion }\end{array}$} & \multirow[t]{2}{*}{$\begin{array}{l}k_{\mathrm{H} 3 \mathrm{O}^{+}} \\
\text {see }^{\text {b) }}\end{array}$} & \multirow[t]{2}{*}{$\begin{array}{l}k_{\mathrm{OH}} \\
\text { see }^{\mathrm{c}}\end{array}$} & \multirow[t]{2}{*}{$\begin{array}{l}Y_{\text {SOA }} \\
\text { see }^{d}\end{array}$} & \multicolumn{5}{|c|}{$\begin{array}{c}\text { Feed composition } \\
\text { (average } \pm 1 \mathrm{SD}), \mathrm{ppmC}\end{array}$} \\
\hline & & & & & & & $\begin{array}{c}\text { E5 } \\
\left(E 6^{*}\right) \\
n=2\end{array}$ & $\begin{array}{c}\mathrm{E} 3 \\
\left(E 4^{*}\right) \\
n=2\end{array}$ & $\begin{array}{l}\text { E7 } \\
n=4\end{array}$ & $\begin{array}{l}\text { E8 } \\
n=2\end{array}$ & $\begin{array}{c}\mathrm{E} 9 \\
\left(E 10^{*}\right) \\
n=2\end{array}$ \\
\hline benzene & mono-c. & 79 & {$\left[\mathrm{C}_{6} \mathrm{H}_{6}+\mathrm{H}\right]^{+}$} & 1.93 & 1.22 & 0.33 & 56 & 109 & 178 & 44 & 234 \\
\hline naphthalene & PAH & 129 & {$\left[\mathrm{C}_{10} \mathrm{H}_{8}+\mathrm{H}\right]^{+}$} & 2.45 & 23 & 0.52 & $\begin{array}{l} \pm 1 \\
11\end{array}$ & $\begin{array}{l} \pm 2 \\
21\end{array}$ & $\begin{array}{l} \pm 5 \\
29\end{array}$ & $\begin{array}{l} \pm 7 \\
8.1\end{array}$ & $\begin{array}{l} \pm 9 \\
40\end{array}$ \\
\hline phenol & phOH & 95 & {$\left[\mathrm{C}_{6} \mathrm{H}_{6} \mathrm{O}+\mathrm{H}\right]^{+}$} & 2.13 & 28 & 0.44 & $\begin{array}{l} \pm 0 \\
9.1\end{array}$ & $\begin{array}{l} \pm 1 \\
13\end{array}$ & $\begin{array}{l} \pm 4 \\
17\end{array}$ & $\begin{array}{c} \pm 1.0 \\
6.4\end{array}$ & $\begin{array}{l} \pm 4 \\
19\end{array}$ \\
\hline toluene & mono-c. & 93 & {$\left[\mathrm{C}_{7} \mathrm{H}_{8}+\mathrm{H}\right]^{+}$} & 2.08 & 5.63 & 0.24 & $\begin{array}{l} \pm 0.6 \\
4.2\end{array}$ & $\begin{array}{l} \pm 0 \\
7.6\end{array}$ & $\begin{array}{l} \pm 1 \\
11\end{array}$ & $\begin{array}{c} \pm 0.7 \\
3.0\end{array}$ & $\begin{array}{l} \pm 1 \\
10\end{array}$ \\
\hline styrene & vinylic & 105 & {$\left[\mathrm{C}_{8} \mathrm{H}_{8}+\mathrm{H}\right.$} & 2.27 & 28 & 0.32 & $\begin{array}{c} \pm 0.4 \\
1.9\end{array}$ & $\begin{array}{l} \pm 0.2 \\
3.5\end{array}$ & $\begin{array}{l} \pm 1 \\
4.9\end{array}$ & $\begin{array}{c} \pm 0.4 \\
1.4\end{array}$ & $\begin{array}{l} \pm 0 \\
3.6\end{array}$ \\
\hline & & & & & & & \pm 0.2 & \pm 0.1 & \pm 0.4 & \pm 0.2 & \pm 0.4 \\
\hline prop-2-enal & $\begin{array}{l}\text { non- } \\
\text { ArHC }\end{array}$ & 57 & {$\left[\mathrm{C}_{3} \mathrm{H}_{4} \mathrm{O}+\mathrm{H}\right]^{+}$} & 3.43 & 20 & 0.02 & $\begin{array}{c}2.7 \\
\pm 0.2\end{array}$ & $\begin{array}{c}3.2 \\
\pm 0.1\end{array}$ & $\begin{array}{c}4.0 \\
\pm 0.2\end{array}$ & $\begin{array}{c}1.2 \\
\pm 0.1\end{array}$ & $\begin{array}{c}3.5 \\
\pm 0.1\end{array}$ \\
\hline benzaldehyde & ox. ArHC & 107 & {$\left[\mathrm{C}_{7} \mathrm{H}_{6} \mathrm{O}+\mathrm{H}\right]^{+}$} & 3.63 & 40 & 0.32 & $\begin{array}{c}1.9 \\
\pm 0.2\end{array}$ & $\begin{array}{c}3.6 \\
\pm 0.1\end{array}$ & $\begin{array}{c}3.4 \\
\pm 0.2\end{array}$ & $\begin{array}{c}1.0 \\
\pm 0.1\end{array}$ & $\begin{array}{c}4.1 \\
\pm 0.1\end{array}$ \\
\hline methylphenol & phOH & 109 & {$\left[\mathrm{C}_{7} \mathrm{H}_{8} \mathrm{O}+\mathrm{H}\right]^{+}$} & 2.27 & $34-48$ & 0.36 & 2.0 & 2.5 & 2.9 & 1.3 & $\begin{array}{l} \pm 0.1 \\
2.5\end{array}$ \\
\hline$(o-/ m-1$ & & & & & & & \pm 0.3 & \pm 0.1 & \pm 0.2 & \pm 0.2 & \pm 0.2 \\
\hline $2-/ 3-m$ & furan & 83 & {$\left[\mathrm{C}_{5} \mathrm{H}_{6} \mathrm{O}+\mathrm{H}\right]^{+}$} & 2 & $62-73$ & 0.07 & $\begin{array}{c}1.1 \\
\pm 0.1\end{array}$ & $\begin{array}{c}0.9 \\
\pm 0.0\end{array}$ & $\begin{array}{c}1.6 \\
\pm 0.1\end{array}$ & $\begin{array}{c}0.5 \\
\pm 0.1\end{array}$ & $\begin{array}{c}0.8 \\
\pm 0.1\end{array}$ \\
\hline acenaphthylene & PAH & 153 & {$\left[\mathrm{C}_{12} \mathrm{H}_{8}+\mathrm{H}\right]^{+}$} & 2.86 & $100-120$ & 0.06 & 0.7 & 1.0 & 1.4 & 0.6 & 1.1 \\
\hline 2-methylprop-2-enal/ & non- & 71 & {$\left[\mathrm{C}_{4} \mathrm{H}_{6} \mathrm{O}+\mathrm{H}\right]^{+}$} & 3.43 & $33-40$ & 0.03 & $\begin{array}{c} \pm 0.3 \\
1.0\end{array}$ & $\begin{array}{c} \pm 0.3 \\
1.0\end{array}$ & $\begin{array}{c} \pm 0.5 \\
1.3\end{array}$ & $\begin{array}{c} \pm 0.2 \\
0.4\end{array}$ & $\begin{array}{c} \pm 0.6 \\
0.8\end{array}$ \\
\hline $\begin{array}{l}\text { (2E)-2-butenal } \\
o \text {-/m-/p-xylene, }\end{array}$ & $\begin{array}{l}\text { ArHC } \\
\text { mono-c. }\end{array}$ & 107 & {$\left[\mathrm{C}_{8} \mathrm{H}_{10}+\mathrm{H}\right]^{+}$} & 2.26 & $7-23$ & 0.20 & $\begin{array}{c} \pm 0.1 \\
1.0\end{array}$ & $\begin{array}{l} \pm 0.0 \\
1.5\end{array}$ & $\begin{array}{l} \pm 0.1 \\
1.0\end{array}$ & $\begin{array}{c} \pm 0.0 \\
0.3\end{array}$ & $\begin{array}{c} \pm 0.1 \\
0.7\end{array}$ \\
\hline $\begin{array}{l}\text { ethylbenzene } \\
\text { 1-/2-methyl- }\end{array}$ & PAH & 143 & {$\left[\mathrm{C}_{11} \mathrm{H}_{10}+\mathrm{H}\right]^{+}$} & 2.71 & 52 & 0.52 & $\begin{array}{c} \pm 0.1 \\
0.7\end{array}$ & $\begin{array}{c} \pm 0.1 \\
1.1\end{array}$ & $\begin{array}{c} \pm 0.1 \\
1.2\end{array}$ & $\begin{array}{l} \pm 0.0 \\
0.4\end{array}$ & $\begin{array}{c} \pm 0.0 \\
0.9\end{array}$ \\
\hline $\begin{array}{l}\text { naphthalene } \\
\text { furan }\end{array}$ & furan & 69 & {$\left[\mathrm{C}_{4} \mathrm{H}_{4} \mathrm{O}+\mathrm{H}\right]^{+}$} & 1.69 & 40 & 0.05 & $\begin{array}{c} \pm 0.1 \\
0.8\end{array}$ & $\begin{array}{c} \pm 0.1 \\
0.7\end{array}$ & $\begin{array}{c} \pm 0.3 \\
1.2\end{array}$ & $\begin{array}{l} \pm 0.0 \\
0.4\end{array}$ & $\begin{array}{c} \pm 0.3 \\
0.6\end{array}$ \\
\hline & & & & & & & \pm 0.1 & \pm 0.0 & \pm 0.2 & \pm 0.0 & \pm 0.1 \\
\hline 2,4-/2,5-dimethyl- & furan & 97 & {$\left[\mathrm{C}_{6} \mathrm{H}_{8} \mathrm{O}+\mathrm{H}\right]^{+}$} & 2 & $87-130$ & 0.32 & 0.9 & 0.6 & 0.8 & 0.3 & 0.4 \\
\hline $\begin{array}{l}\text { furan } \\
2,4-/ 2,6-/ 3,5- \\
\text { dimethylphenol (xy }\end{array}$ & $\mathrm{phOH}$ & 123 & {$\left[\mathrm{C}_{8} \mathrm{H}_{10} \mathrm{O}+\mathrm{H}\right]^{+}$} & 2 & $80-91$ & 0.26 & $\begin{array}{c} \pm 0.1 \\
0.6 \\
\pm 0.1\end{array}$ & $\begin{array}{c} \pm 0.0 \\
0.6 \\
\pm 0.1\end{array}$ & $\begin{array}{c} \pm 0.1 \\
0.6\end{array}$ & $\begin{array}{c} \pm 0.0 \\
0.3 \\
+0.3\end{array}$ & $\begin{array}{c} \pm 0.0 \\
0.4 \\
+0.4\end{array}$ \\
\hline & phOH & 111 & {$\left[\mathrm{C}_{6} \mathrm{H}_{6} \mathrm{O}_{2}+\mathrm{H}\right]^{+}$} & 2 & 104 & 0.39 & $\begin{array}{c} \pm 0.1 \\
0.6 \\
\pm 0.1\end{array}$ & $\begin{array}{l}M .1 \\
0.5 \\
\pm 0.0\end{array}$ & $\begin{array}{c}7.1 \\
0.6 \\
\pm 0.1\end{array}$ & $\begin{array}{c} \pm 0.1 \\
0.3 \\
\pm 0.0\end{array}$ & $\begin{array}{c} \pm .1 \\
0.4 \\
\pm 0.1\end{array}$ \\
\hline 1,2-dihydroacenaphthylene & PAH & 155 & {$\left[\mathrm{C}_{12} \mathrm{H}_{10}+\mathrm{H}\right]^{+}$} & 2.81 & $7-8$ & 0.07 & 0.4 & 0.5 & 0.6 & 0.2 & 0.5 \\
\hline $\begin{array}{l}\left(1,1^{\prime} \text {-biphenyl) }\right. \\
\text { 2-methoxyphenol }\end{array}$ & phOH & 125 & {$\left[\mathrm{C}_{7} \mathrm{H}_{8} \mathrm{O}_{2}+\mathrm{H}\right]^{+}$} & 2 & $54-78$ & 0.45 & $\begin{array}{c} \pm 0.1 \\
0.3\end{array}$ & $\begin{array}{c} \pm 0.0 \\
0.2\end{array}$ & $\begin{array}{c} \pm 0.2 \\
0.4\end{array}$ & $\begin{array}{c} \pm 0.1 \\
0.2\end{array}$ & $\begin{array}{c} \pm 0.3 \\
0.2\end{array}$ \\
\hline 1,2-dimethyl- & PAH & 157 & {$\left[\mathrm{C}_{12} \mathrm{H}_{12}+\mathrm{H}\right]^{+}$} & 2 & 77 & 0.31 & $\begin{array}{c} \pm 0.1 \\
0.2\end{array}$ & $\begin{array}{c} \pm 0.0 \\
0.2\end{array}$ & $\begin{array}{c} \pm 0.1 \\
0.1\end{array}$ & $\begin{array}{c} \pm 0.0 \\
0.0\end{array}$ & $\begin{array}{c} \pm 0.0 \\
0.1\end{array}$ \\
\hline $\begin{array}{l}\text { naphthalene } \\
\text { 4-(2-hydroxyethyl)phenol/ }\end{array}$ & $\mathrm{phOH}$ & 139 & {$\left[\mathrm{C}_{8} \mathrm{H}_{10} \mathrm{O}_{2}+\mathrm{H}\right]^{+}$} & 2 & 75 & 0.32 & $\begin{array}{c} \pm 0.1 \\
0.1\end{array}$ & $\begin{array}{c} \pm 0.0 \\
0.1\end{array}$ & $\begin{array}{c} \pm 0.0 \\
0.2\end{array}$ & $\begin{array}{c} \pm 0.0 \\
0.1\end{array}$ & $\begin{array}{c} \pm 0.0 \\
0.1\end{array}$ \\
\hline & $\mathrm{phOH}$ & & & & & & \pm 0.0 & \pm 0.0 & \pm 0.0 & \pm 0.0 & $\begin{array}{c} \pm 0.0 \\
0.0\end{array}$ \\
\hline & & (1) & {$\left[\mathrm{C}_{8} \mathrm{H}_{10} \mathrm{O}_{3}+\mathrm{H}\right]$} & 2 & $75-81$ & 0.26 & $\begin{array}{c}0.1 \\
\pm 0.0 \\
\end{array}$ & \pm 0.0 & \pm 0.1 & $\begin{array}{c}0.1 \\
\pm 0.0 \\
\end{array}$ & $\begin{array}{c}0.0 \\
\pm 0.0 \\
\end{array}$ \\
\hline "SOA-22 precursor" & & & & & & & $97 \pm 5$ & $173 \pm 1$ & $261 \pm 4$ & $71 \pm 10$ & $324 \pm 1$ \\
\hline NMOC (PTR-ToF-MS) & & & & & & & $179 \pm 15$ & $265 \pm 13$ & $381 \pm 28$ & $110 \pm 14$ & $404 \pm 7$ \\
\hline NMHC (FID) & & & & & & & 90 & 160 & 260 & 105 & 250 \\
\hline NMHC (FID-CRDS) & & & & & & & 95 & 160 & 250 & 105 & 250 \\
\hline 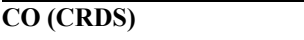 & & & & & 0.15 & & 450 & 700 & 1000 & 400 & 1100 \\
\hline $\mathrm{CH}_{4}$ (CRDS) & & & & & 0.00635 & & 80 & 130 & 200 & 75 & 220 \\
\hline
\end{tabular}

E3-E10 denote different experiments (Table 1). *E6 and *E4, *E10 are OFR experiments; the relative composition within those corresponds to E5 and E3, E9 conducted within the same experimental set. Compound classes: mono-c.=monocyclic ArHC), $\mathrm{PAH}=$ polycyclic $\mathrm{ArHC}, \mathrm{phOH}=$ phenolic $\mathrm{ArHC}$, non-ArHC=aliphatic compounds, furan=furans, ox. ArHC=oxygenated ArHC other than phenolic compounds. ${ }^{\circ} \mathrm{NMOC}=$ non-methane organic compounds. Note that concentration levels refer to the feed for the catalytic converters; emissions were diluted at least 8-fold before fed to instrumentation/OFR, see set-up in Figure S1. ${ }^{\text {a) }}$ Ions are referred to with their integer mass-to-charge ratio $(\mathrm{m} / \mathrm{z})$ for simplicity, but are identified based on the exact $m / z$. ${ }^{\text {b) }}$ proton transfer reaction rate constant ${ }^{5,9}, k_{\mathrm{H} 3 \mathrm{O}}{ }^{+}, 10^{-9} \mathrm{~cm}^{3} \mathrm{~s}^{-1} .{ }^{\mathrm{c})}$ atmospheric hydroxyl radical rate constants ${ }^{56-64}, k_{\mathrm{OH}}, 10^{-12} \mathrm{~cm}^{3} \mathrm{molecules}^{-1} \mathrm{~s}^{-}$ ${ }^{1}$ (range in brackets corresponds to isomers and spread in reported data). ${ }^{\text {d) }}$ SOA yield, $Y_{\text {SOA }}$, ( $\mu$ g SOA per $\mu$ g reacted SOA precursor) applied here based on ref. .

In order to obtain further information about our mass closure and the validity of using the FIDbased NMHC signal as a predictor for SOA-22 precursors, we compared the PTR-ToF-MS measurements to the FID-based NMHC signal. The ratio of PTR-ToF-MS-to-FID was $1.6 \pm 0.3$ 
ppmC:ppmC considering the complete PTR-ToFMS carbon mass. Both instruments are selective towards specific compound classes, and an obtained ratio may thus be close to 1 due to compensating errors. While oxygenated compounds with short carbon (as were observed by the PTR-ToF-MS, e.g. methanol) have limited response on the FID, aliphatic compounds are detected only selectively ${ }^{7}$ by PTR-ToF-MS. This is elaborated further in the SI (section S3).

SOA formation. SOA formation was studied experimentally via exposure of the emissions to hydroxyl radicals in an OFR. We assessed the contributions of the individual SOA-22 species to the OFR reacted gaseous precursor mass (Figure $3 b$ ), and predicted a corresponding SOA-22 particle phase mass (Figure 3c).

The largest contributors to the OFR reacted SOA-22 precursor mass were phenol (22-28\%), naphthalene (21-29\%) and benzene (6-29\%) This results from species' abundance becoming essentially weighted by their corresponding atmospheric reactivity upon OFR photochemistry $\left(k_{\mathrm{OH}}\right.$, in units of $10^{-12} \mathrm{~cm}^{3}$ molecules $\mathrm{s}^{-1}$, Table 2). Among these three species, $k_{\mathrm{OH}}$ is highest for phenol (28), followed by naphthalene (23), and lowest for benzene (1.22). Therefore, contributions of phenol and naphthalene increased relative to benzene in Figure $3 \mathrm{~b}$.

In order to predict the SOA-22 particle phase mass ("predicted SOA-22 SOA), the OFR reacted SOA-22 precursor mass was weighted with compound-specific SOA yields $\left(Y_{\mathrm{SOA}}\right)$ from literature, relying on the evaluation of this approach by ref. ${ }^{9}$. Details and a discussion of its limitations are provided in section S3 and Eq. S1. The SOA yields describe the fraction of reacted SOA precursor that condenses into the particle phase. $Y_{\mathrm{SOA}}$ for the three main species is 0.44 (phenol), 0.52 (naphthalene) and 0.33 (benzene) as summarized in Table 2 . The value of the experimentally determined particle phase SOA mass of fresh emissions was $1.20 \pm 0.81$ times that of the predicted value (summing all estimated SOA contributions). For catalytically cleaned emissions, the ratio was $1.35 \pm 0.73$. The method therefore underestimated the experimentally determined particle phase SOA by $20 \%$ and $35 \%$ on average.
Benzene, despite dominating the gas-phase emissions by accounting for $58-72 \%$ of the SOA22 precursor mass (Figure 3a), finally contributed relatively less to the predicted SOA mass in the particle phase (5-25\%), owing to its comparatively lower atmospheric reactivity. Instead, naphthalene and phenol together were responsible for a substantial fraction $(55-71 \%)$ of the predicted SOA in the OFR experiments, including 30-38\% derived from naphthalene and $25-33 \%$ from phenol (Figure $3 \mathrm{c}$ /Figure S7). An additional 3-7\% of the SOA formation was attributed to cresol, $2-6 \%$ to styrene and $2-3 \%$ to benzaldehyde. Catalytic abatement of phenolic compounds and naphthalene should therefore substantially reduce the observed SOA mass, as we confirm in the next section.

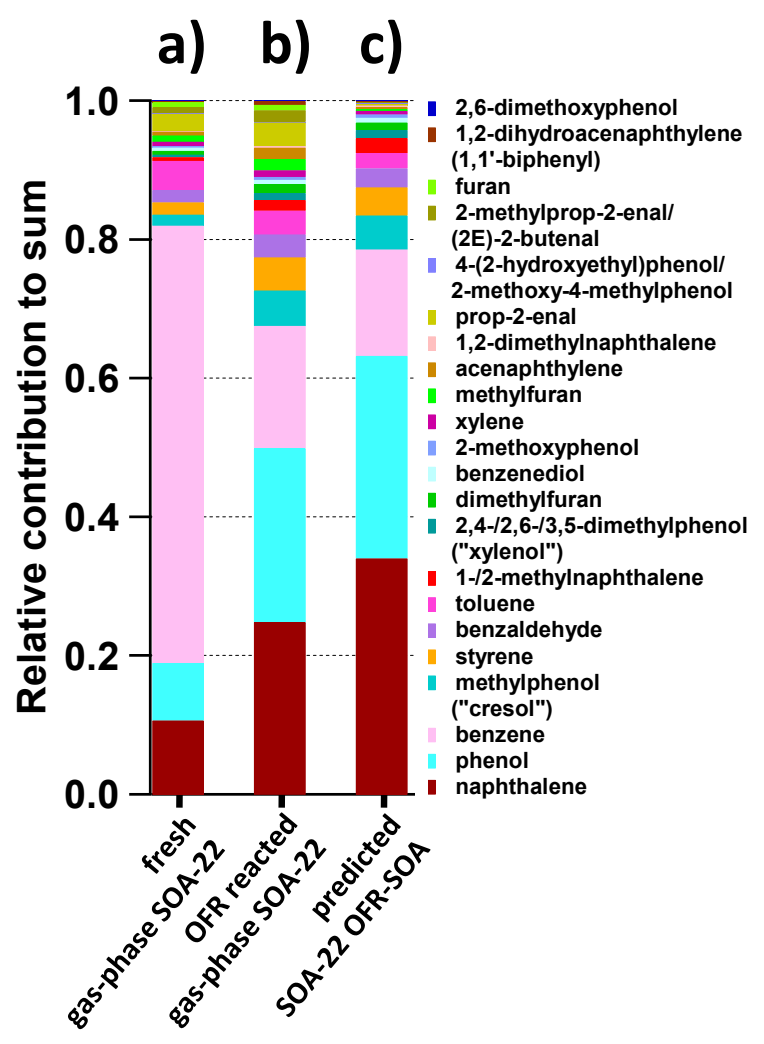

Figure 3. Relative contributions of $\operatorname{SOA}-22\left(\mu \mathrm{g} \mathrm{m}^{-3}\right)$. a) fresh gas-phase composition based on the PTR-ToF-MS sum of SOA-22 (experiments E5/E6 and E3/E4, E7, E8 and E9/E10), b) photo-chemically aged, i.e. OFR reacted gasphase (E6, E4, E10), c) "predicted SOA-22 SOA" relying on published SOA yields ${ }^{9}$ (E6, E4, E10). 
SOA reduction. Figure 4 presents the effect of a catalytic converter on the SOA formation. We observed a substantial reduction of SOA formation upon catalytic cleaning of the emissions. As the fraction of converted NMHC and SOA-22 precursors increased with catalyst temperature, SOA formation decreased. In the case of $\mathrm{Pt} / \mathrm{Al}_{2} \mathrm{O}_{3}$, SOA was significantly reduced at temperatures as low as $185^{\circ} \mathrm{C}$ (by $68 \%$ ). For $\mathrm{Pt} / \mathrm{CeO}_{2}-\mathrm{Al}_{2} \mathrm{O}_{3}$, SOA reduction was almost complete $(>97 \%)$ at $310^{\circ} \mathrm{C}$, and likewise significant at the lower temperatures, reaching $62 \%$ reduction at $185^{\circ} \mathrm{C}$.

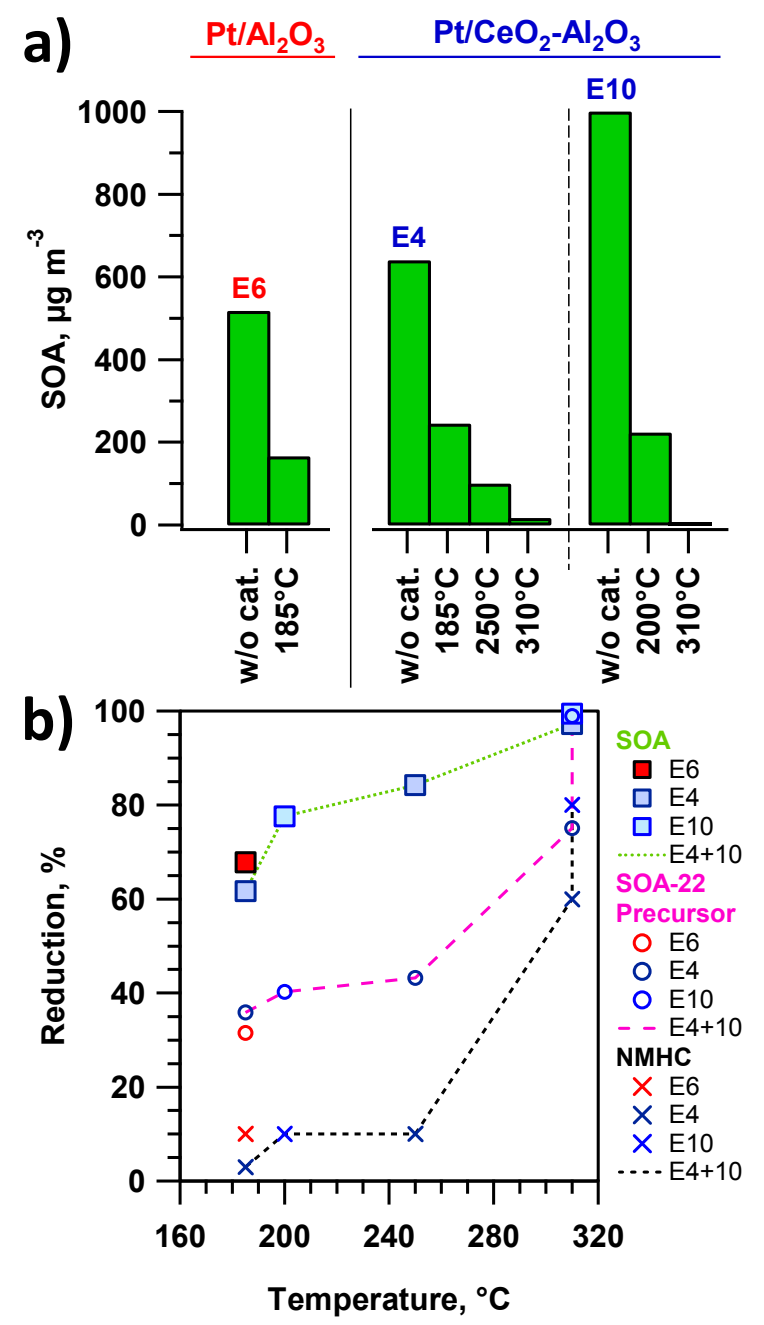

Figure 4. Secondary organic aerosol (SOA) formation. a) Fresh emissions (i.e. without catalyst, "w/o cat.") and emissions cleaned at specific temperature set-points of the catalytic monoliths are presented. $\mathrm{Pt} / \mathrm{Al}_{2} \mathrm{O}_{3}$ at $185^{\circ} \mathrm{C}$ (E6), $\mathrm{Pt} / \mathrm{CeO}_{2}-\mathrm{Al}_{2} \mathrm{O}_{3}$ at 185 to $310^{\circ} \mathrm{C}$ (E4 and E10). Fresh emission SOA was measured in duplicate (average $\pm 1 \mathrm{SD}$ are $519 \pm 3$ (E6), $641 \pm 20$ (E4), $\left.1001 \pm 15(\mathrm{E} 10) \mu \mathrm{g} \mathrm{m}^{-3}\right)$, b) SOA reduction, NMHC reduction and "SOA-22 precursor" reduction of catalytically cleaned compared to fresh emissions.
The experimentally measured SOA reduction was linked to a) NMHC reduction, b) SOA-22 precursor reduction and c) reduction of "predicted SOA-22 SOA" in Figure 5 (Figure S8 gives further details). The SOA reduction coincided with the removal of only a small fraction of the NMHC, such as 3 to $10 \%$ of the FID-based NMHC signal below $185^{\circ} \mathrm{C}$, and 60 to $80 \%$ at $310^{\circ} \mathrm{C}$. At this temperature SOA was almost completely removed (Figure 5a). The NMHC signal by itself is therefore an insufficient predictor for SOA mass reduction. Instead, the summed SOA-22 precursor reduction describes the measured SOA reduction better (Figure $5 b$ ).

Compounds within the SOA-22 sum contribute to the SOA mass at distinct fractions that differ from their gas-phase contributions. As detailed in the previous section, these fractions are a function of 1) the precursors' abundance, 2) the actual OFR reacted mass (i.e., essentially corresponding to the compounds' abundance weighted by its $k_{\mathrm{OH}}$ ), and 3) the SOA yield. Therefore, only a comparison of the experimentally measured SOA reduction with the reduction of "predicted SOA-22 SOA" yields a relationship close to 1:1 (Figure 5c). Partitioning effects ${ }^{65-67}$ play an additional role in the observed SOA reduction assessment and add uncertainty to our analysis (see our discussion in SI section S3, Table S2 and Figure S9 for further information).

A substantial fraction (55-71\%) of the SOA mass is attributed to naphthalene and phenol. Although their complete removal would reduce the SOA-22 precursor mass only by $17-21 \%$, a particle phase SOA-22 SOA mass reduction of $55-71 \%$ would be achieved. Indeed, phenol and naphthalene are significantly abated by the catalytic converters. At low catalyst temperatures, a particular impact on the SOA reduction may be assigned to the effective removal of phenolic compounds, given that at $185^{\circ} \mathrm{C}$, their abatement was $>95 \%$, while naphthalene abatement remained below 35\% (Figure 2). While the removal of $95 \%$ of phenol results only in a SOA-22 precursor reduction of 6$9 \%$, a reduction of $24-31 \%$ of the predicted SOA22 SOA mass can be achieved. Naphthalene abatement by $35 \%$ instead translates into a SOA-22 precursor reduction of $\sim 4 \%$ and a corresponding predicted SOA mass reduction of $11-13 \%$. 


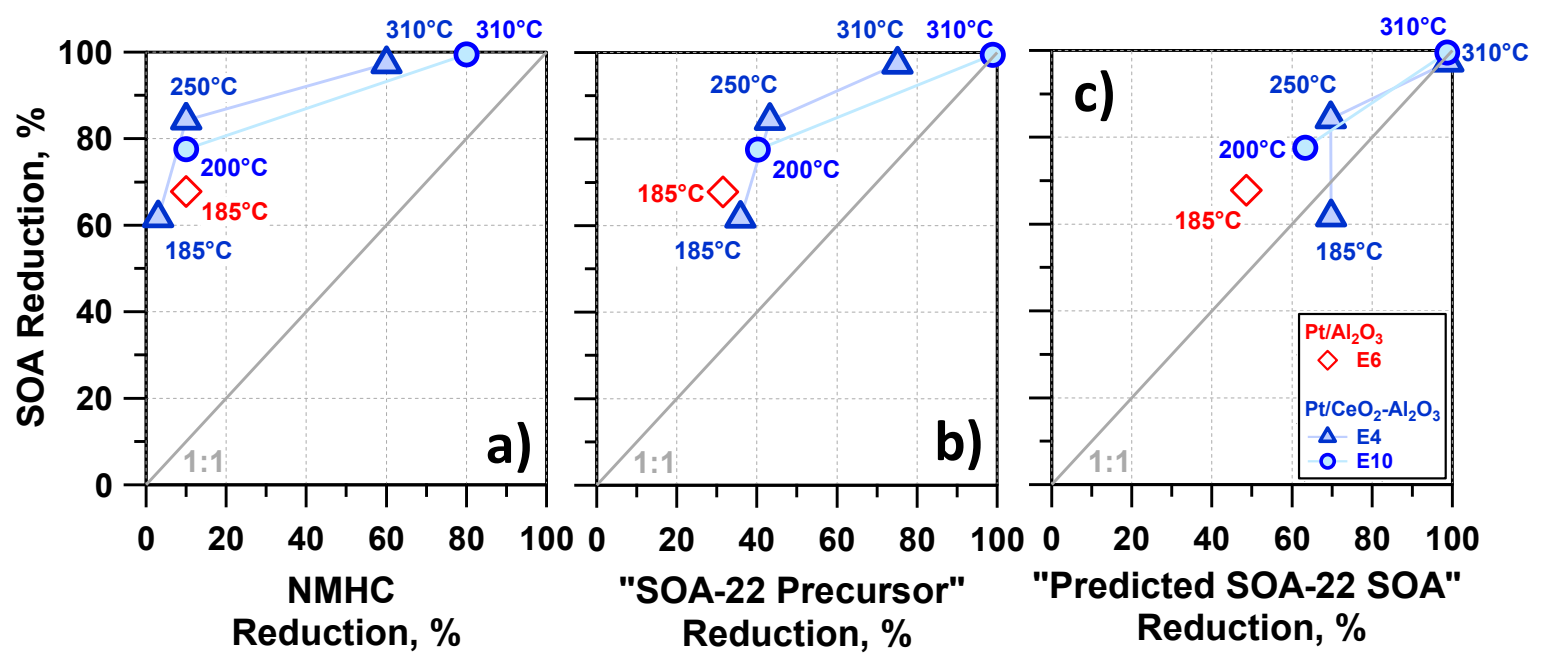

Figure 5. Secondary organic aerosol (SOA) reduction. a) SOA reduction vs. NMHC reduction. NMHC is derived from Figure 1, as it is not directly accessible in E6, E4 and E10. See Figure S6 for CO conversion points). b) SOA reduction vs." SOA-22 precursor" reduction, c) SOA reduction vs. "predicted SOA-22 SOA" reduction Figure S8 presents additionally FID-based NMHC vs. PTR-ToF-MS-based "SOA-22 precursor" reduction, and "SOA-22 Precursor" vs. "Predicted SOA-22 SOA" reduction.

In sum, the SOA-22 precursor reduction is $10-13 \%$ and the expected SOA reduction $35-45 \%$. Instead, removing $10-13 \%$ benzene from the SOA-22 precursors would result in maximal SOA mass reduction of $10 \%$. Effective removal of atmospherically reactive compounds with high SOA yields at the lower catalyst temperatures is therefore a crucial aspect in the SOA reduction. It results in a gradually less efficient precursor composition in terms of SOA formation (lower $k_{\mathrm{OH}}$ and lower yield) as a function of catalyst temperature.

\section{Implications}

Our results demonstrate the effective removal of critical emissions constituents such as toxic $\mathrm{CO}$ and harmful aromatic hydrocarbons, using catalytic converters. This significantly lowers the health impact as well as the SOA formation potential of wood burning emissions. Through reduced SOA formation, the total particulate matter pollution can be reduced substantially considering the high $\mathrm{POA} / \mathrm{BC}$ and high SOA/POA ratios reported for $\log$ wood burning. ${ }^{6}, 9$ Low catalyst light-off temperatures are crucial, because appliances emit large fractions of VOC and harmful aromatic compounds during start-up phases, re-fueling and burn-out, when the exhaust temperature is relatively low, and when also stove automatization has limited effects to reduce emissions. $3,20,25,27,28$,
${ }^{68}$ Both, CO and SOA precursors, are effectively removed at sufficiently low temperatures in our experiments, supporting also the application of cheaper catalytic materials than $\mathrm{Pt}$ in the future. Instead, the reduction of methane, which should be abated owing to its high global warming potential, requires tailored catalysts. $\mathrm{CeO}_{2}$ has the potential to lower the required conversion temperatures. Alternately, installation in the burner chamber could be considered to reach higher temperatures, but catalyst stability may become an even bigger challenge under such conditions. This requires attention in research and development projects. The overall technical applicability (such as clogging and issues with pressure drops) remains to be investigated. Installations of catalytic converters downstream of an electrostatic precipitator ${ }^{27}$ may be an effective strategy to minimize catalysts' exposure to particulate matter for long-term operation. Also engineering optimization allowing for higher pressure drops, such as optimized dimensions and the use of flue gas fans, offer to overcome technical constraints and may support long-term operation for catalytic gas cleaning systems as presented here, and potentially also the catalytic removal of primary particulate matter presented elsewhere ${ }^{31,35-37}$. 


\section{Acknowledgements}

This work was funded by the Competence Center Environment and Sustainability (project OPTIWARES) and we would like to thank our collaborators within this project for their valuable contributions to the scientific discussion. This work was also supported by the Swiss National Science projects WOOSHI (No. 140590) and NRP 70 ("Energy Turnaround") and the European Community's Seventh Framework Programme (FP7/2007-2013) under grant agreement no. 290605 (PSI-FELLOW). U.B. acknowledges additional funding from the European Union's Horizon 2020 research and innovation programme through the EUROCHAMP-2020 Infrastructure Activity under grant agreement no. 730997. We thank René Richter for his invaluable technical assistance.

\section{Supporting Information}

Supporting Information is available as noted in the text. This material is available free of charge via the Internet.

\section{References}

1. Bari, M. A.; Baumbach, G.; Kuch, B.; Scheffknecht, G., Particle-phase concentrations of polycyclic aromatic hydrocarbons in ambient air of rural residential areas in southern Germany. Air Qual Atmos Health 2010, 3, (2), 103-116.

2. Querol, X.; Alastuey, A.; Ruiz, C. R.; Artiñano, B.; Hansson, H. C.; Harrison, R. M.; Buringh, E.; ten Brink, H. M.; Lutz, M.; Bruckmann, P.; Straehl, P.; Schneider, J., Speciation and origin of PM10 and PM2.5 in selected European cities. Atmos. Environ. 2004, 38, (38), 6547-6555.

3. Schmidl, C.; Luisser, M.; Padouvas, E.; Lasselsberger, L.; Rzaca, M.; Ramirez-Santa Cruz, C.; Handler, M.; Peng, G.; Bauer, H.; Puxbaum, H., Particulate and gaseous emissions from manually and automatically fired small scale combustion systems. Atmos. Environ. 2011, 45, (39), 7443-7454.

4. Bruns, E. A.; Krapf, M.; Orasche, J.; Huang, Y.; Zimmermann, R.; Drinovec, L.; Močnik, G.; ElHaddad, I.; Slowik, J. G.; Dommen, J.; Baltensperger, U.; Prévôt, A. S. H., Characterization of primary and secondary wood combustion products generated under different burner loads. Atmos. Chem. Phys. 2015, 15, (5), 2825-2841.

5. Bruns, E. A.; Slowik, J. G.; El Haddad, I.; Kilic, D.; Klein, F.; Dommen, J.; Temime-Roussel, B.; Marchand, N.; Baltensperger, U.; Prévôt, A. S. H., Characterization of gas-phase organics using proton transfer reaction time-of-flight mass spectrometry: fresh and aged residential wood combustion emissions. Atmos. Chem. Phys. 2017, 17, (1), 705-720.

6. Bertrand, A.; Stefenelli, G.; Bruns, E. A.; Pieber, S. M.; Temime-Roussel, B.; Slowik, J. G.; Prévôt, A. S. H.; Wortham, H.; El Haddad, I.; Marchand, N., Primary emissions and secondary aerosol production potential from woodstoves for residential heating: Influence of the stove technology and combustion efficiency. Atmos. Environ. 2017, 169, 6579.

7. Grieshop, A. P.; Logue, J. M.; Donahue, N. M.; Robinson, A. L., Laboratory investigation of photochemical oxidation of organic aerosol from wood fires 1: measurement and simulation of organic aerosol evolution. Atmos. Chem. Phys. 2009, 9, (4), 1263-1277.

8. Heringa, M. F.; DeCarlo, P. F.; Chirico, R.; Lauber, A.; Doberer, A.; Good, J.; Nussbaumer, T.; Keller, A.; Burtscher, H.; Richard, A.; Miljevic, B.; Prévôt, A. S. H.; Baltensperger, U., Time-resolved characterization of primary emissions from residential wood combustion appliances. Environ. Sci. Technol. 2012, 46, (20), 11418-11425.

9. Bruns, E. A.; El Haddad, I.; Slowik, J. G.; Kilic, D.; Klein, F.; Baltensperger, U.; Prévôt, A. S. H., Identification of significant precursor gases of secondary organic aerosols from residential wood combustion. Sci Rep 2016, 6, 27881.

10. Integrated Risk Information System (IRIS). U.S. Environmental Protection Agency: Washington, DC. https://www.epa.gov/iris (July 20, 2017),

11. Agency for Toxic Substances and Diseases Registry (ATSDR): Substances A-Z. U.S. Environmental Protection Agency: Washington, DC. http://www.atsdr.cdc.gov/substances/indexAZ.asp (July 20, 2017),

12. Huang, Z.-H.; Zhang, Y.-L.; Yan, Q.; Wang, Z.-Y.; Zhang, Z.; Wang, X.-M., Decreased Human Respiratory Absorption Factors of Aromatic Hydrocarbons at Lower Exposure Levels: The Dual Effect in Reducing Ambient Air Toxics. Environmental Science \& Technology Letters 2017, 4, (11), 463-469.

13. Platt, S. M.; El Haddad, I.; Zardini, A. A.; Clairotte, M.; Astorga, C.; Wolf, R.; Slowik, J. G.; Temime-Roussel, B.; Marchand, N.; Ježek, I.; Drinovec, L.; Močnik, G.; Möhler, O.; Richter, R.; Barmet, P.; Bianchi, F.; Baltensperger, U.; Prévôt, A. S. H., Secondary organic aerosol formation from gasoline vehicle emissions in a new mobile environmental reaction chamber. Atmos. Chem. Phys. 2013, 13, (18), 9141-9158.

14. Platt, S. M.; El Haddad, I.; Pieber, S. M.; Zardini, A. A.; Suarez-Bertoa, R.; Clairotte, M.; Daellenbach, K. R.; Huang, R. J.; Slowik, J. G.; Hellebust, S.; Temime-Roussel, B.; Marchand, N.; de Gouw, J.; Jimenez, J. L.; Hayes, P. L.; Robinson, A. L.; Baltensperger, U.; Astorga, C.; Prévôt, A. S. H., Gasoline cars produce more carbonaceous particulate matter than modern filter-equipped diesel cars. Sci Rep 2017, 7, (1), 4926.

15. Pieber, S. M.; Kumar, N. K.; Klein, F.; Comte, P.; Bhattu, D.; Dommen, J.; Bruns, E. A.; Kılıç, D.; El 
Haddad, I.; Keller, A.; Czerwinski, J.; Heeb, N.; Baltensperger, U.; Slowik, J. G.; Prévôt, A. S. H., Gasphase composition and secondary organic aerosol formation from standard and particle filter-retrofitted gasoline direct injection vehicles investigated in a batch and flow reactor. Atmos. Chem. Phys. 2018, 18, (13), 9929-9954.

16. Molteni, U.; Bianchi, F.; Klein, F.; El Haddad, I.; Frege, C.; Rossi, M. J.; Dommen, J.; Baltensperger, U., Formation of highly oxygenated organic molecules from aromatic compounds. Atmos. Chem. Phys. 2018, 18, (3), 1909-1921.

17. Schwantes, R. H.; Schilling, K. A.; McVay, R. C.; Lignell, H.; Coggon, M. M.; Zhang, X.; Wennberg, P. O.; Seinfeld, J. H., Formation of highly oxygenated low-volatility products from cresol oxidation. Atmos. Chem. Phys. 2017, 17, (5), 3453-3474.

18. Boman, C.; Pettersson, E.; Westerholm, R.; Boström, D.; Nordin, A., Stove Performance and Emission Characteristics in Residential Wood Log and Pellet Combustion, Part 1: Pellet Stoves. Energy \& Fuels 2011, 25, 307-314.

19. Johansson, L. S.; Leckner, B.; Gustavsson, L.; Cooper, D.; Tullin, C.; Potter, A., Emission characteristics of modern and old-type residential boilers fired with wood logs and wood pellets. Atmos. Environ. 2004, 38, (25), 4183-4195.

20. Pettersson, E. r.; Boman, C.; Westerholm, R.; Boström, D.; Nordin, A., Stove Performance and Emission Characteristics in Residential Wood Log and Pellet Combustion, Part 2: Wood Stove. Energy \& Fuels 2011, 25, (1), 315-323.

21. Bianchi, F.; Barmet, P.; Stirnweis, L.; El Haddad, I.; Platt, S. M.; Saurer, M.; Lötscher, C.; Siegwolf, R.; Bigi, A.; Hoyle, C. R.; DeCarlo, P. F.; Slowik, J. G.; Prévôt, A. S. H.; Baltensperger, U.; Dommen, J., Contribution of methane to aerosol carbon mass. Atmos. Environ. 2016, 141, 41-47.

22. Wöhler, M.; Andersen, J. S.; Becker, G.; Persson, H.; Reichert, G.; Schön, C.; Schmidl, C.; Jaeger, D.; Pelz, S. K., Investigation of real life operation of biomass room heating appliances - Results of a European survey. Appl. Energy 2016, 169, 240-249. 23. Reichert, G.; Schmidl, C.; Haslinger, W.; Schwabl, M.; Moser, W.; Aigenbauer, S.; Wöhler, M.; Hochenauer, C., Investigation of user behavior and assessment of typical operation mode for different types of firewood room heating appliances in Austria. Renewable Energy 2016, 93, 245-254.

24. Fachinger, F.; Drewnick, F.; Gieré, R.; Borrmann, S., How the user can influence particulate emissions from residential wood and pellet stoves: Emission factors for different fuels and burning conditions. Atmos. Environ. 2017, 158, 216-226.

25. Czech, H.; Pieber, S. M.; Tiitta, P.; Sippula, O.; Kortelainen, M.; Lamberg, H.; Grigonyte, J.; Streibel, T.; Prévôt, A. S. H.; Jokiniemi, J.; Zimmermann, R., Time-resolved analysis of primary volatile emissions and secondary aerosol formation potential from a smallscale pellet boiler. Atmos. Environ. 2017, 158, 236-245.

26. Ward, T. J.; Palmer, C. P.; Bergauff, M.; Jayanty, R. K. M.; Noonan, C. W., Organic/elemental carbon and woodsmoke tracer concentrations following a community wide woodstove changeout program. Atmos. Environ. 2011, 45, (31), 5554-5560.

27. Bindig, R.; Hartmann, I.; Koch, C.; Matthes, M.; Schenker, M.; Thiel, C.; Kraus, M.; Roland, U.; Einicke, W.-D., Abgasreinigung an Biomassekleinfeuerungsanlagen und experimentelle Untersuchungen zur Kombination von katalytischen und elektrostatischen Abgasreinigungsverfahren. Chemie Ingenieur Technik 2011, 83, (12), 2105-2120.

28. Reichert, G.; Schmidl, C.; Haslinger, W.; Stressler, H.; Sturmlechner, R.; Schwabl, M.; Wöhler, M.; Hochenauer, C., Catalytic efficiency of oxidizing honeycomb catalysts integrated in firewood stoves evaluated by a novel measuring methodology under reallife operating conditions. Renewable Energy 2018, 117, 300-313.

29. Bindig, R.; Butt, S.; Hartmann, I.; Matthes, M.; Thiel, C., Application of Heterogeneous Catalysis in Small-Scale Biomass Combustion Systems. Catalysts 2012, 2, (4), 223-243.

30. Ozil, F.; Tschamber, V.; Haas, F.; Trouvé, G., Efficiency of catalytic processes for the reduction of $\mathrm{CO}$ and VOC emissions from wood combustion in domestic fireplaces. Fuel Processing Technology 2009, 90, (9), 1053-1061.

31. Hukkanen, A.; Kaivosoja, T.; Sippula, O.; Nuutinen, K.; Jokiniemi, J.; Tissari, J., Reduction of gaseous and particulate emissions from small-scale wood combustion with a catalytic combustor. Atmos. Environ. 2012, 50, 16-23.

32. Bensaid, S.; Deorsola, F. A.; Fino, D.; Russo, N., After-treatment of household wood-fired stove emissions: From catalyst formulation to full-scale system. Catalysis Today 2012, 197, (1), 76-89.

33. Carno, J., Catalytic abatement of emissions from small-scale combustion of wood: A comparison of the catalytic effect in model and real flue gases. Fuel 1996, 75, (8), 959-965.

34. Ferrandon, M.; Berg, M.; Björnbom, E., Thermal stability of metal-supported catalysts for reduction of cold-start emissions in a wood-fired domestic boiler. Catalysis Today 1999, 53, (4), 647-659. 35. Shromova, O. A.; Kinnunen, N. M.; Pakkanen, T. A.; Suvanto, M., Promotion effect of water in catalytic fireplace soot oxidation over silver and platinum. RSC Adv. 2017, 7, (73), 46051-46059.

36. Wöhler, M.; Jaeger, D.; Pelz, S. K.; Thorwarth, H., Potential of Integrated Emissions Reduction Systems in a Firewood Stove under Real Life Operation Conditions. Energy \& Fuels 2017, 31, (7), 7562-7571.

37. Kaivosoja, T.; Viren, A.; Tissari, J.; Ruuskanen, J.; Tarhanen, J.; Sippula, O.; Jokiniemi, J., Effects of a catalytic converter on PCDD/F, chlorophenol and $\mathrm{PAH}$ emissions in residential wood combustion. Chemosphere 2012, 88, (3), 278-85.

38. Bruns, E. A.; El Haddad, I.; Keller, A.; Klein, F.; Kumar, N. K.; Pieber, S. M.; Corbin, J. C.; Slowik, J. G.; Brune, W. H.; Baltensperger, U.; Prévôt, A. S. H., Inter-comparison of laboratory smog chamber and flow reactor systems on organic aerosol yield and 
composition. Atmos. Meas. Tech. 2015, 8, (6), 23152332 .

39. Kleemann, M.; Elsener, M.; Koebel, M.; Wokaun, A., Investigation of the ammonia adsorption on monolithic SCR catalysts by transient response analysis. Appl. Catal. B Environ. 2000, 27, (4), 231-242. 40. Reece, S. M.; Sinha, A.; Grieshop, A. P., Primary and Photochemically Aged Aerosol Emissions from Biomass Cookstoves: Chemical and Physical Characterization. Environ Sci Technol 2017, 51, (16), 9379-9390.

41. Barmet, P.; Dommen, J.; DeCarlo, P. F.; Tritscher, T.; Praplan, A. P.; Platt, S. M.; Prévôt, A. S. H.; Donahue, N. M.; Baltensperger, U., OH clock determination by proton transfer reaction mass spectrometry at an environmental chamber. Atmos. Meas. Tech. 2012, 5, (3), 647-656.

42. Finlayson-Pitts, B. J.; Pitts, J. N., Chemistry of the Upper and Lower Atmosphere. Theory, Experiments, and Applications. Academic Press: 2000; p 969.

43. Peng, Z.; Day, D. A.; Ortega, A. M.; Palm, B. B.; Hu, W.; Stark, H.; Li, R.; Tsigaridis, K.; Brune, W. H.; Jimenez, J. L., Non-OH chemistry in oxidation flow reactors for the study of atmospheric chemistry systematically examined by modeling. Atmos. Chem. Phys. 2016, 16, (7), 4283-4305.

44. Peng, Z.; Day, D. A.; Stark, H.; Li, R.; LeeTaylor, J.; Palm, B. B.; Brune, W. H.; Jimenez, J. L., HOx radical chemistry in oxidation flow reactors with low-pressure mercury lamps systematically examined by modeling. Atmos. Meas. Tech. 2015, 8, (11), 4863-4890.

45. Peng, Z.; Jimenez, J. L., Modeling of the chemistry in oxidation flow reactors with high initial NO. Atmos. Chem. Phys. 2017, 17, (19), 11991-12010.

46. Li, R.; Palm, B. B.; Ortega, A. M.; Hlywiak, J.; Hu, W.; Peng, Z.; Day, D. A.; Knote, C.; Brune, W. H.; de Gouw, J. A.; Jimenez, J. L., Modeling the radical chemistry in an oxidation flow reactor: radical formation and recycling, sensitivities, and the $\mathrm{OH}$ exposure estimation equation. J Phys Chem A 2015, 119, (19), 4418-32.

47. Palm, B. B.; Campuzano-Jost, P.; Ortega, A. M.; Day, D. A.; Kaser, L.; Jud, W.; Karl, T.; Hansel, A.; Hunter, J. F.; Cross, E. S.; Kroll, J. H.; Peng, Z.; Brune, W. H.; Jimenez, J. L., In situ secondary organic aerosol formation from ambient pine forest air using an oxidation flow reactor. Atmos. Chem. Phys. 2016, 16, (5), 2943-2970.

48. Jordan, A.; Haidacher, S.; Hanel, G.; Hartungen, E.; Märk, L.; Seehauser, H.; Schottkowsky, R.; Sulzer, P.; Märk, T. D., A high resolution and high sensitivity proton-transfer-reaction time-of-flight mass spectrometer (PTR-TOF-MS). Int. J. Mass Spectrom. 2009, 286, (2-3), 122-128.

49. Graus, M.; Muller, M.; Hansel, A., High resolution PTR-TOF: Quantification and formula confirmation of VOC in real time. Journal of the American Society for Mass Spectrometry 2010, 21, 1037--1044.

50. DeCarlo, P. F.; Kimmel, J. R.; Trimborn, A.; Northway, M. J.; Jayne, J. T.; Aiken, A. C.; Gonin, M.; Fuhrer, K.; Horvath, T.; Docherty, K. S.; Worsnop, D.
R.; Jimenez, J. L., Field-deployable, high-resolution, time-of-flight aerosol mass spectrometer. Anal. Chem. 2006, 78, (24), 8281-8289.

51. $\quad$ Canagaratna, M. R.; Jayne, J. T.; Jimenez, J. L.; Allan, J. D.; Alfarra, M. R.; Zhang, Q.; Onasch, T. B.; Drewnick, F.; Coe, H.; Middlebrook, A., Chemical and microphysical characterization of ambient aerosols with the Aerodyne aerosol mass spectrometer. Mass Spectrom Rev 2007, 26, (2), 185-222.

52. Pieber, S. M.; El Haddad, I.; Slowik, J. G.; Canagaratna, M. R.; Jayne, J. T.; Platt, S. M.; Bozzetti, C.; Daellenbach, K. R.; Frohlich, R.; Vlachou, A.; Klein, F.; Dommen, J.; Miljevic, B.; Jimenez, J. L.; Worsnop, D. R.; Baltensperger, U.; Prévôt, A. S. H., Inorganic Salt Interference on $\mathrm{CO}_{2}{ }^{+}$in Aerodyne AMS and ACSM Organic Aerosol Composition Studies. Environ Sci Technol 2016, 50, (19), 10494-10503.

53. Gélin, P.; Primet, M., Complete oxidation of methane at low temperature over noble metal based catalysts: a review. Appl. Catal. B Environ. 2002, 39, (1), 1-37.

54. Trovarelli, A.; de Leitenburg, C.; Boaro, M.; Dolcetti, G., The utilization of ceria in industrial catalysis. Catalysis Today 1999, 50, (2), 353-367.

55. Marchionni, V.; Szlachetko, J.; Nachtegaal, M.; Kambolis, A.; Krocher, O.; Ferri, D., An operando emission spectroscopy study of $\mathrm{Pt} / \mathrm{Al} 2 \mathrm{O} 3$ and Pt/CeO2/A12O3. Phys Chem Chem Phys 2016, 18, (42), 29268-29277.

56. Atkinson, R.; Arey, J., Atmospheric degradation of volatile organic compounds. Chem Rev 2003, 103, (12), 4605-38.

57. Jenkin, M. E.; Saunders, S. M.; Wagner, V.; Pilling, M. J., Protocol for the development of the Master Chemical Mechanism, MCM v3 (Part B): tropospheric degradation of aromatic volatile organic compounds. Atmos. Chem. Phys. 2003, 3, 181-193.

58. Bloss, C.; Wagner, V.; Jenkin, M. E.; Volkamer, R.; Bloss, W. J.; Lee, J. D.; Heard, D. E.; Wirtz, K.; Martin-Reviejo, M.; Rea, G.; Wenger, J. C.; Pilling, M. J., Development of a detailed chemical mechanism (MCMv3.1) for the atmospheric oxidation of aromatic hydrocarbons. Atmos. Chem. Phys. 2005, 5, 641-664.

59. Olariu, R. I.; Barnes, I.; Becker, K. H.; Klotz, B., Rate coefficients for the gas-phase reaction of $\mathrm{OH}$ radicals with selected dihydroxybenzenes and benzoquinones. International Journal of Chemical Kinetics 2000, 697-702.

60. Atkinson, R.; Aschmann, S. M., Kinetics of the reactions of naphthalene, 2-methylnaphthalene, and 2,3dimethylnaphthalene with $\mathrm{OH}$ radicals and with $\mathrm{O}_{3}$ at $295 \pm 1$ K. International Journal of Chemical Kinetics 1986, 18, (5), 569-573.

61. Lauraguais, A.; Bejan, I.; Barnes, I.; Wiesen, P.; Coeur, C., Rate Coefficients for the Gas-Phase Reactions of Hydroxyl Radicals with a Series of Methoxylated Aromatic Compounds. The Journal of Physical Chemistry A 2015, 119, (24), 6179-6187.

62. Ullerstam, M.; Ljungström, E.; Langer, S., Reactions of acrolein, crotonaldehyde and pivalaldehyde with $\mathrm{Cl}$ atoms: structure-activity relationship and 
comparison with $\mathrm{OH}$ and $\mathrm{NO} 3$ reactions. Physical Chemistry Chemical Physics 2001, 3, (6), 986-992.

63. Orlando, J. J.; Tyndall, G. S., Mechanisms for the Reactions of $\mathrm{OH}$ with Two Unsaturated Aldehydes: Crotonaldehyde and Acrolein. The Journal of Physical Chemistry A 2002, 106, (51), 12252-12259.

64. NIST Chemical Kinetics Database. National Institute of Standards and Technology. https://kinetics.nist.gov/kinetics/KineticsSearchForm.jsp (December 22, 2018),

65. Pankow, J. F., An Absorption-Model of GasParticle Partitioning of Organic-Compounds in the Atmosphere. Atmos. Environ. 1994, 28, (2), 185-188.

66. Odum, J. R.; Hoffmann, T.; Bowman, F.; Collins, D.; Flagan, R. C.; Seinfeld, J. H., Gas/particle partitioning and secondary organic aerosol yields. Environ. Sci. Technol. 1996, 30, (8), 2580-2585.

67. Donahue, N. M.; Robinson, A. L.; Stanier, C. O.; Pandis, S. N., Coupled partitioning, dilution, and chemical aging of semivolatile organics. Environ Sci Technol 2006, 40, (8), 2635-43.

68. Elsasser, M.; Busch, C.; Orasche, J.; Schön, C.; Hartmann, H.; Schnelle-Kreis, J.; Zimmermann, R., Dynamic Changes of the Aerosol Composition and Concentration during Different Burning Phases of Wood Combustion. Energy \& Fuels 2013, 27, (8), 4959-4968. 